\title{
¿CANDIDATAS O ESPECTADORAS? UN ANÁLISIS DEL IMPACTO DE LA LEY DE CUOTAS
}

\author{
Joaquín Galeno \\ Pontificia Universidad Católica de Chile \\ Francisco Gallego \\ Pontificia Universidad Católica de Chile \\ Felipe González \\ Pontificia Universidad Católica de Chile
}

\begin{abstract}
Resumen: Este artículo expone que, luego de la introducción de la ley de cuotas, los partidos políticos nominaron a candidatas mujeres en peores condiciones que a candidatos hombres en las elecciones parlamentarias del año 2017. Así, ellas tuvieron 20 puntos porcentuales menos de probabilidad de ocupar el primer lugar de su partido en la papeleta y recibieron un millón de pesos menos en aportes de su colectividad. Los cálculos realizados indican que la desventaja en la ubicación en la papeleta electoral se transformó en una pérdida que
\end{abstract}

JoAQuín GALENO. Ingeniero comercial y magíster en economía por la Pontificia Universidad Católica de Chile. Email: jagaleno@uc.cl

Francisco Gallego. PhD por el Instituto Tecnológico de Massachusetts (MIT). Profesor de economía de la Pontificia Universidad Católica de Chile. Email: fgallego@, uc.cl.

Felipe GonzÁlez. PhD en economía por la UC Berkeley. Profesor de economía de la Pontificia Universidad Católica de Chile. Email: fagonza4@gmail.com.

Los autores agradecen los comentarios y las sugerencias de Felipe Aldunate, Vittorio Corbo, José Díaz, Andrés Hojman, Jeanne Lafortune, Rolf Lüders, José Tessada y Gert Wagner, así como de los árbitros anónimos de Estudios Públicos. También agradecen al proyecto Fondecyt regular 1170956 por el financiamiento entregado. 
va de una a cinco parlamentarias. El artículo termina discutiendo potenciales diseños alternativos que afinarían la política de cuotas y los desafíos que implicarían.

Palabras Clave: cuotas de género, elecciones parlamentarias, papeleta, posicionamiento, candidatas mujeres.

RECIBIDO: marzo 2019; ACEPTADO: mayo 2019.

Clasificación JEL: D72; K16.

\section{CANDIDATES OR ONLOOKERS? AN ANALYSIS OF THE IMPACT OF THE QUOTA LAW}

ABSTRACT: This article shows that following the introduction of the quota law, political parties nominated female candidates on worse terms than male candidates in the 2017 parliamentary elections. Thus, women were 20 percentage points less likely to be first-placed for their party on the ballot paper and received a million pesos less funding from it. The calculations carried out indicate that this poor ballot placement translated into a loss of between one and five women parliamentarians. The article concludes by discussing potential alternative designs that would refine quota policy, and the challenges these would entail.

KEYWORDs: gender quotas, parliamentary elections, ballot, positioning, female candidates.

RECEIVED: March 2019; ACCEPTED: May 2019.

JEL Classification: D72; K16.

\section{INTRODUCCIÓN}

a menor participación femenina en espacios de representación política y de poder ha sido una preocupación cada vez mayor dentro del debate público y académico. Esto porque, a pesar de que las mujeres conforman aproximadamente el 50 por ciento de la población, al año 2015 sólo el 12 por ciento de los escaños legislativos disponibles a nivel mundial eran ocupados por representantes del género femenino (Kanthak y Woon 2015). Aquello ha sido reconocido por gobiernos y la sociedad civil en diferentes países, lo que ha llevado a impulsar mecanismos de acción afirmativa que tienen por objetivo aumentar esta proporción. La más común de estas medidas es la implementación de cuotas de género, las que consisten en la obligación de contar con un número determinado de candidatas mujeres a nivel de partidos en las 
elecciones en que éstos participan. En la actualidad, más de 100 países han adoptado en alguna medida este tipo de política dentro de sus sistemas de representación (Internacional IDEA et al. 2018), ya sea de forma obligatoria (a través de la ley electoral) o voluntaria (a través de los reglamentos internos de los partidos).

Chile, desde el año 2015, también se sumó a este grupo de países a través de la promulgación de la Ley 20.840, que reforma su sistema electoral, pasando de un sistema binominal a uno proporcional "moderado". En esta ley se establece que ningún género podrá superar el 60 por ciento del total de los candidatos inscritos por los partidos a nivel nacional — de lo contrario, toda la lista quedará invalidada_-, a la vez que entrega incentivos monetarios para la inclusión de mujeres a través de una mayor devolución de gastos de campaña.

A pesar de los esfuerzos por incrementar la participación política femenina, la efectividad de las cuotas de género es variada entre países. En el caso chileno, luego de que se introdujo la cuota, la participación de representantes femeninas en la Cámara de Diputados se ha visto incrementada en 6,7 puntos porcentuales (desde una base de 15,8), mientras que para el Senado este aumento fue de 7,4 puntos porcentuales (partiendo de igual base). Si bien este incremento es mayor a la tendencia de aumento de representación femenina que se venía registrando en las elecciones parlamentarias chilenas, resulta relativamente bajo en comparación con la experiencia de otros países que han implementado este tipo de política.

La figura 1 muestra una selección de países en los que se han incorporado diversos tipos de cuotas de género. La cuota chilena presenta una baja efectividad en relación con legislaciones similares en otros países. Mientras un incremento de un punto porcentual de cuota en el caso argentino logró un aumento de 0,76 puntos porcentuales en la participación de mujeres en el parlamento, para Chile este efecto alcanza sólo 0,28 puntos porcentuales. Las distintas formas de aplicar las cuotas entre países pueden ser una causa importante detrás de esta variación. ${ }^{1}$

${ }^{1}$ Si bien podría sugerirse la existencia de efectos no lineales en la cuota, de manera que la efectividad dependa del porcentaje inicial de mujeres en el parlamento, en los datos se evidencia que mientras Suecia presenta una alta efectividad partiendo desde una base más alta (36 por ciento) que el caso chileno, Argentina también logra un nivel alto de logro en el objetivo de esta política partiendo de un nivel inicial menor ( 5 por ciento de participación femenina en el Congreso) a la participación femenina exhibida en Chile. 
Figura 1. COMPARACIÓN DEL IMPACTO DE LA LEY DE CUOTAS ENTRE PAÍSES

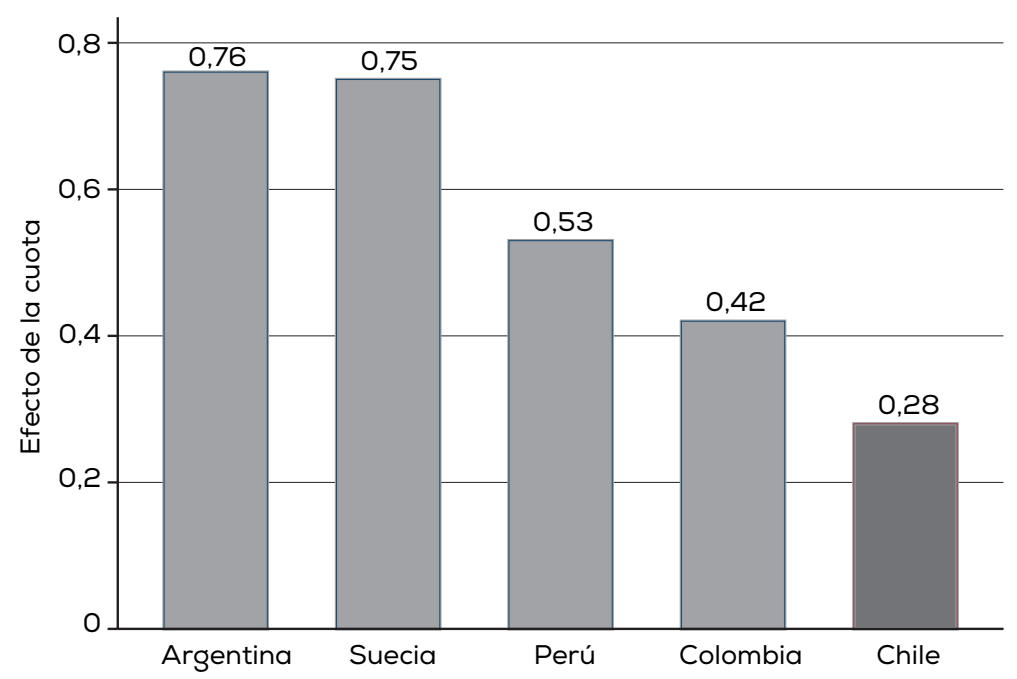

Nota: Los efectos son calculados para la elección previa y posterior a la incorporación de la cuota. Los cálculos para cada país en esta figura se obtienen dividiendo (i) la diferencia entre el porcentaje de mujeres en el parlamento antes y después de la introducción de la cuota de género, con (ii) la diferencia entre el porcentaje de la cuota establecida y el porcentaje de mujeres en el parlamento previo a la incorporación de la cuota.

¿Qué elementos de la legislación que regula la competencia electoral podrían explicar la mayor efectividad de unas legislaciones sobre las otras? El trabajo de Schwindt-Bayer (2009) muestra que si bien las cuotas tienen un impacto positivo a la hora de aumentar la participación femenina en las cámaras legislativas, su efecto se ve disminuido a un tercio si es que no están acompañadas de (i) mecanismos que aseguren su cumplimiento y (ii) de mandatos acerca de la localización de los candidatos en la papeleta. ${ }^{2} \mathrm{Si}$ bien la autora logra cuantificar este menor impacto, no identifica cuál es el rol que está jugando cada una de estas características de las cuotas en el comportamiento de la participación

${ }^{2}$ Ejemplos de estos mandatos son lo que se ha conocido como zipper cuota, en los cuales los candidatos se deben alternar por sexo dentro de la papeleta, o mecanismos más débiles, en los cuales sólo los dos primeros candidatos deben ser de géneros distintos. 
femenina parlamentaria. Por otro lado, Esteve-Volart y Bagues (2012) muestran el rol que tienen los partidos para afectar el resultado electoral de las candidatas al poner en los primeros lugares de la papeleta de votación a los candidatos masculinos, lo que va en línea de lo encontrado por Besley et al. (2017) respecto al rol de los líderes del partido en no incorporar mujeres competentes debido a la preocupación de que puedan ser reemplazados producto del aumento de competencia interna.

Esto motiva el objetivo de este artículo. Tomando en consideración que en la última elección parlamentaria los partidos chilenos cumplieron con la cuota, ${ }^{3}$ esta investigación busca revisar mecanismos que los partidos pueden utilizar para mermar las posibilidades de las mujeres de ser electas y que podrían estar jugando un rol en la efectividad de la ley en la última elección parlamentaria. Este artículo estudia tres razones que podrían explicar la baja efectividad de las cuotas en el caso chileno: el aporte monetario a las candidaturas, la posición dentro de la papeleta y la elección de la zona electoral en las cuales compiten los candidatos.

Los resultados indican que las candidatas tuvieron 20 puntos porcentuales menos de probabilidad de encabezar la lista de candidatos de sus partidos. $\mathrm{Y}$ es conocido que existen efectos significativos en la votación producto del posicionamiento de los postulantes dentro de la papeleta electoral, algo que se ha denominado ballot-order effect: nominados que ocupan las primeras posiciones tienen ganancias en votación de entre 2 y 6 puntos porcentuales. ${ }^{4} \mathrm{Al}$ mismo tiempo, las candidatas al parlamento en 2017 recibieron cerca de un millón de pesos menos de parte de sus partidos, lo que logra explicar un tercio de la brecha que existe entre hombres y mujeres. Finalmente, no encontramos evidencia respecto a que los partidos nominen a menos mujeres en las zonas electorales en las que son más competitivos ni de mayor tamaño.

${ }^{3}$ Existen tres partidos, entre 25, que terminaron con un menor porcentaje que el 40 por ciento establecido, pero esto se explica por rechazos en las candidaturas enviadas inicialmente. De igual forma, el no cumplimiento observado por estos partidos corresponde a un máximo de dos candidatas menos a las requeridas.

${ }^{4}$ La figura 2 muestra la distribución de las candidaturas a nivel de partidos (electas y no electas), según género. Como se puede apreciar, los partidos posicionan a las mujeres en lugares "más abajo" dentro de la cédula electoral que la contraparte masculina; del mismo modo, se muestra que quienes se posicionan más arriba logran la elección con mayor regularidad. 
Figura 2. POSICIONAMIENTO DE CANDIDATOS (H) Y CANDIDATAS (M) EN LA PAPELETA ELECTORAL, CHILE 2017

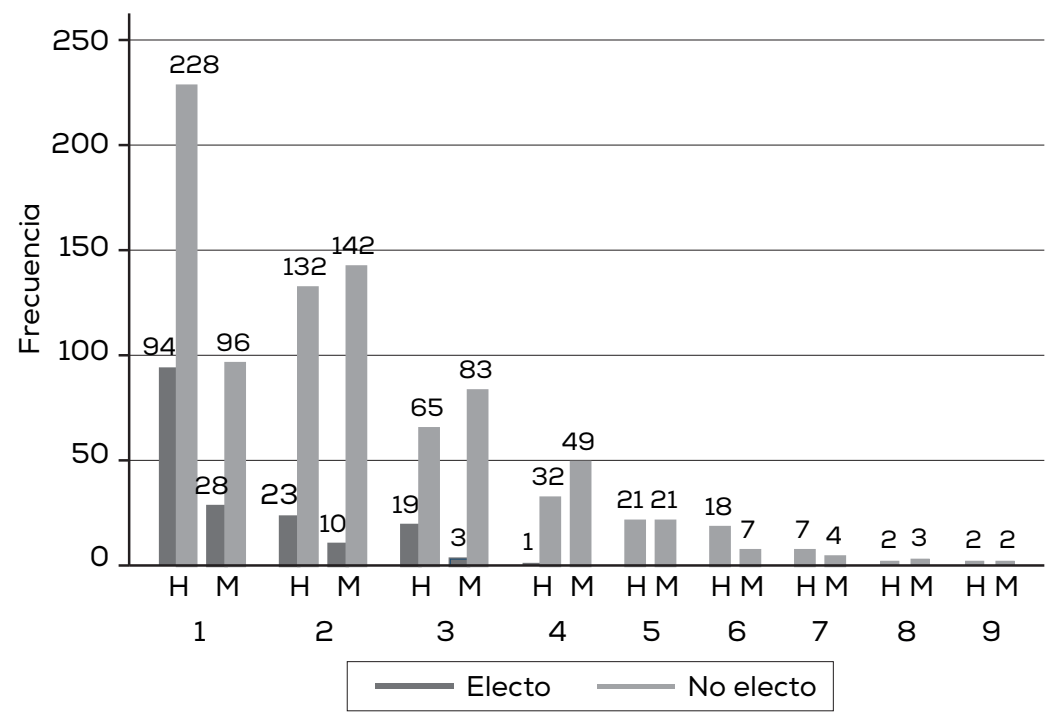

Nota: Este gráfico utiliza datos de las elecciones parlamentarias del 2017 en Chile. El eje $x$ corresponde a la posición en la papeleta electoral (1ra-9na posición). El color de las barras distingue si el candidato ganó o perdió la elección. El eje y mide el número de candidatos en cada categoría.

El artículo termina por evaluar el impacto de distintos ordenamientos de los candidatos según género dentro de la papeleta electoral. Usando simulaciones de ordenamientos alternativos encontramos que el número de mujeres parlamentarias aumentaría entre uno y cinco si las mujeres encabezaran la papeleta o el orden de los candidatos fuera aleatorio. Esto corresponde a una reducción de hasta 10 por ciento (8 puntos porcentuales) de la brecha calculada para Chile en relación con los países que han logrado mayor efectividad en la implementación de las cuotas.

\section{LITERATURA RELACIONADA}

Este trabajo se suma a la literatura que estudia la participación femenina en política y las cuotas de género. Existen varios trabajos que han intentado comprender los efectos de la inclusión de este tipo de 
intervención tanto en variables observables relevantes como en políticas desarrolladas una vez que se incorporan mujeres en los espacios de poder.

En el primer grupo, Baltrunaite et al. (2014), por ejemplo, muestran que la incorporación de cuotas de género aumentó la cantidad de años de educación en los candidatos electos y que esto se debe no sólo a la elección de mujeres más educadas, sino que además se dejan de elegir a los hombres menos educados. Asimismo, Bagues y Campa (2017) presentan evidencia de que las cuotas para municipalidades pequeñas llevaron aparejadas un aumento en la cantidad de mujeres electas, no así en la calidad de los políticos electos, medida a través de la educación promedio de éstos. Por el lado de los sesgos en los votantes, De Paola et al. (2010) muestran que las cuotas son efectivas para reducir el sesgo negativo que existe dentro de la población respecto de las candidatas, aumentando la participación de mujeres en las administraciones locales aun después de haber retirado las cuotas en el sistema de elección. Finalmente, por el lado de la representación, Miranda y SuárezCao (2018) estudian el rol de las mujeres en la política chilena, con un capítulo especial dedicado al desempeño electoral de las mujeres en las elecciones parlamentarias de 2017, y Schwindt-Bayer (2018) presenta estudios del rol de las mujeres en la política latinoamericana de manera más general.

Dentro del segundo grupo de trabajos, que abordan políticas desarrolladas una vez que se incorporan mujeres en los espacios de poder, encontramos a Clots-Figueras (2011), investigación que muestra cómo legisladoras mujeres en cupos reservados pueden cambiar políticas orientadas a favorecer a las mujeres, así como también tiene un impacto positivo en políticas redistributivas. En la misma línea, Beaman et al. (2009) muestran que la exposición a cuotas reduce los sesgos negativos hacia las mujeres y que la presencia de asientos reservados en espacios de liderazgo aumenta la probabilidad de que mujeres compitan y ganen en las elecciones del consejo de villa en India. Además de esto, también encuentran una mejora en las percepciones del liderazgo femenino y un debilitamiento de los estereotipos de género en el espacio público y privado.

La importancia de ambos grupos de trabajos radica en que entregan evidencia acerca de la capacidad de las cuotas de género en conseguir 
sus objetivos, así como documentan las consecuencias que conlleva la implementación de cuotas auténticamente efectivas. En esta línea, Caminotti y Freidenberg (2016) estudian la efectividad de las cuotas en Argentina y México, y concluyen que es necesario prestar atención al comportamiento de los partidos a la hora de las nominaciones.

Por otro lado, una tercera línea de investigación busca introducir elementos adicionales a las explicaciones de oferta y demanda tradicionales que se han hecho a las políticas de acción afirmativa. En particular, intenta entregar evidencia acerca del comportamiento de los partidos políticos luego de la introducción de una ley de cuotas. En este grupo de trabajos podemos encontrar a Besley et al. (2017), quienes muestran el rol que tienen los líderes de partidos en Suecia para dificultar la entrada de mujeres, puesto que esto aumenta la competencia en el mercado de líderes políticos (o líderes de partidos), lo que termina por aumentar la probabilidad de que pierdan el poder. Frechette et al. (2008) investigan desde la perspectiva de los incumbentes el cómo existen diferentes incentivos — que además cambian según el sistema electoral en el que se desarrolle la votación - a la hora de aprobar la incorporación de este tipo de medida, ya que para la aprobación de este tipo de legislación se necesita, obviamente, el voto de individuos que luego competirán. Finalmente, Esteve-Bolart y Bagues (2012) muestran cómo los partidos nominan en peores posiciones a candidatas mujeres, aun en contextos donde el orden de los candidatos es determinado por orden alfabético de los apellidos, evidenciando que los partidos ocupan efectivamente todos los grados de libertad que tienen de forma estratégica.

Todas estas investigaciones muestran que el funcionamiento de las cuotas puede estar influido por comportamientos que van más allá de la contextualización clásica de oferta y demanda que entiende las cuotas como un desvío de la nominación óptima de candidatos, puesto que muestran que los actores involucrados pueden tener incentivos diversos, tanto a la hora de aprobar este tipo de legislación como de competir bajo ella. Esto es particularmente importante, dado que esta investigación busca mostrar canales alternativos vía los cuales los partidos responden a las cuotas de género.

Finalmente, la importancia de estudiar el orden de las candidatas dentro del voto se basa en la literatura conocida como ballot-order 
effect; es decir, el efecto causal en votación producto de encabezar la lista en el voto. Esta literatura muestra las ventajas que adquieren las candidatas que ocupan el primer lugar dentro de la papeleta electoral. Por ejemplo, Miller y Krosnick (1998) estiman que pasar a la primera posición de la papeleta entrega 2,5 puntos porcentuales más de votos en el contexto de elecciones en Ohio en 1992. De la misma manera, en América Latina, Gulzar y Ruiz (2018) usan datos de loterías en dos elecciones en Colombia en el año 1974, y encuentran efectos de 6,6 puntos porcentuales en votación producto de la posición, siendo, a nuestro saber, los resultados más altos encontrados. ${ }^{5}$

La literatura desarrollada en este sentido es relevante para esta investigación, puesto que, si bien existe evidencia consistente que muestra la presencia de ballot-order effect en las elecciones, enfatiza que éste depende del nivel de información que tienen los votantes. Tomando en consideración esto para el contexto chileno, se debe entonces tener en cuenta que el cambio de sistema electoral ha producido un aumento en el número de candidatos entre los cuales las personas tendrán que tomar una decisión. Al mismo tiempo, las zonas electorales han aumentado su tamaño, por lo que los candidatos deben abarcar una mayor extensión en su campaña. Todo esto genera una disminución en la cantidad de información asimilable por los votantes.

\section{CONTEXTO}

\subsection{Reforma electoral del 2015}

Bajo el gobierno de Michelle Bachelet, se publicó la Ley 20.840, con la que se reforma el sistema electoral bajo el cual se celebran las elecciones parlamentarias. En esta ley no sólo se incorporaron cuotas de género, sino que también se reformó el sistema electoral parlamentario, la composición de las zonas electorales y se entregaron incentivos a los partidos y a las mujeres para participar en la competencia por asientos en el Congreso.

${ }^{5}$ El trabajo seminal en esta literatura es de Gold (1952). Otros trabajos importantes son los de Koppell y Steen (2004), Ho e Imai (2006) y King y Leigh (2009). El trabajo de Ho e Imai enfatiza que encabezar la lista puede ser más importante cuando los votantes tienen menos información sobre las candidatas. 
De esta forma, se estableció que el sistema para elegir a los parlamentarios consiste en uno que es proporcional "moderado", bajo el método D'Hondt, y en el cual los partidos pueden nominar un total de $n+1$ candidatos en los 28 distritos para los diputados y en 15 circunscripciones electorales para los senadores, donde $n$ corresponde al total de escaños a elegir en la zona electoral. Este número varía entre $3 \mathrm{y}$ 8 diputados y entre 2 y 5 senadores, según el tamaño de la población comprendida en el territorio electoral respectivo. Previo a esto, Chile estaba dividido en 60 distritos y 19 circunscripciones que elegían a 120 diputados y 38 senadores, dos por cada zona electoral correspondiente. Estos eran votados bajo un sistema de elección binominal, en el cual las listas estaban conformadas por dos nominados, que por lo general correspondían a candidatos de distintos partidos.

En relación con las políticas de género implementadas, la nueva ley detalla que ninguno de los dos géneros puede superar el 60 por ciento del total de los candidatos nominados inicialmente a nivel nacional por el partido, lo que en la práctica corresponde a una cuota de género para las mujeres de 40 por ciento, toda vez que han sido ellas las que históricamente no han logrado cumplir con este porcentaje de nominación. Adicionalmente, la ley también establece mecanismos para incentivar la incorporación de mujeres en las elecciones, tanto a nivel de partidos, como a nivel de candidatos. A nivel de partidos, estos serán compensados con 500 UF por cada una de sus candidatas mujeres electas, dinero que podrán ocupar únicamente para actividades que busquen promover la inclusión y participación femenina en política. Del mismo modo, la ley establece que candidatos y partidos tendrán una devolución adicional de 0,01 UF por voto obtenido para el caso de las candidatas. Esto corresponde a un aumento de 25 por ciento en la devolución de gastos por voto en relación con los candidatos masculinos. ${ }^{6}$

Tanto la cuota de género incorporada, como los incentivos monetarios que se detallan en esta sección son válidos únicamente para las elecciones parlamentarias. Para el caso de los consejeros regionales,

${ }^{6}$ Los hombres reciben una devolución de 0,04 UF por voto, mientras que las mujeres, 0,05 UF, lo que equivale a 1.040 y 1.300 pesos, aproximadamente, para cada caso. 
en elecciones que se celebran de forma simultánea a los comicios parlamentarios, estos son electos también bajo el método proporcional D’Hondt en las 64 circunscripciones provinciales del país. ${ }^{7}$

\subsection{Nominación de candidatas}

En lo que respecta a las candidaturas y su posición en la cédula electoral, el Servicio Electoral de Chile (Servel) sortea, para el caso de las elecciones parlamentarias y consejeros regionales, únicamente el orden de aparición de las listas en la cédula. El orden de los partidos dentro de una misma lista lo define el propio pacto, mientras que el orden de las candidatas de un mismo partido es elección del respectivo partido.

Los partidos entonces eligen libremente el orden en que pondrán a cada uno de sus candidatos, si éste será hombre o mujer, dónde le hará competir y cuánto dinero le entregará como aporte a su campaña, sujeto a la cuota de género que debe cumplir. De esta forma, podemos reconocer que, si bien las cuotas de género han restringido los grados de libertad que tienen los partidos políticos a la hora de nominar a sus candidatos y han asegurado la participación femenina en la elección, éstos aún mantienen altos niveles de discrecionalidad al momento de disponer de sus nominados a través de los distintos mecanismos estudiados en esta investigación y que, en la práctica, reducen la participación efectiva que las candidatas pueden llegar a tener en las elecciones.

\section{DATOS}

Nuestro análisis usa datos a nivel de candidatos en las elecciones parlamentarias y de consejeros regionales en los años 2013 y 2017. La base de datos incluye información para todos los distritos (diputados),

${ }^{7}$ Además, el año 2016 se promulga la Ley 20.900, con la que se regula el financiamiento de las candidaturas en busca de una mayor transparencia acerca de los donantes, así como también limitando los montos con los que los candidatos pueden contar para financiar sus campañas. La ley mandata que todos los aportes a las campañas deben ser realizados a través del Servicio Electoral de Chile, el cual publica los montos y el tipo de aporte en su página web. Estas donaciones pueden tener tres fuentes: los aportes entregados por terceros (personas naturales), los partidos y los aportes que los mismos candidatos hacen a sus campañas. Sólo los últimos están sujetos a devoluciones de gasto. 
circunscripciones electorales (senadores) y circunscripciones provinciales (consejeros regionales) del país. Se ha reunido información acerca de la afiliación política, sexo, aportes recibidos a la campaña (desde los partidos, terceros y aportes propios), posición en la cédula electoral y resultado para todos los candidatos. Para los candidatos parlamentarios de la elección de 2017 se cuenta también con medidas de experiencia política, edad y competitividad del partido que representan. En el año 2013 compitieron 91 mujeres y 379 hombres por escaños en la Cámara de Diputados, mientras que en el año 2017 compitieron 397 mujeres y 563 hombres.

Esta información se ha obtenido de dos fuentes. La primera de ellas es información pública de elecciones dispuesta por el Servel, en donde se puede encontrar la información acerca del nombre de los candidatos, la edad, la posición en la papeleta y la zona electoral en la cual están participando, tanto para las votaciones del 2013 como de 2017. La segunda de ellas es a través de la búsqueda en internet de información acerca de la experiencia legislativa que tienen los candidatos. Esta fue dividida en tres variables: (i) si el nominado es incumbente en la elección; (ii) el número de años en el parlamento, en caso de que el candidato haya participado en el poder legislativo anteriormente, y (iii) el número de años en cargos de exposición pública dentro del aparato estatal (alcaldía, gobernación, intendencia, ministerio, etcétera).

El cuadro 1 muestra las características de los candidatos para la elección de 2017. Tanto para senadores como para diputados, los candidatos hombres muestran mayor experiencia política, ya sea a través de más años en cargos legislativos u otros cargos de exposición pública, o son con mayor probabilidad incumbentes que disputan una reelección. Del mismo modo, están situados con mayor probabilidad en los primeros lugares del voto y obtienen también más votación. Así, como esta mayor experiencia política podría estar explicando las ventajas a la hora de competir en las elecciones exhibidas por los hombres respecto de las mujeres, se ocuparán estas variables como controles, de manera de reducir el problema de selección existente en este contexto.

\section{EL COMPORTAMIENTO DE LOS PARTIDOS POLÍTICOS}

Como hemos discutido antes, los partidos políticos contaron con una serie de mecanismos para entregar ventajas a sus candidatos hombres por sobre las mujeres que nominaron el año 2017. En este artículo 
Cuadro 1. ESTADÍSTICAS DESCRIPTIVAS PARA LAS ELECCIONES
PARLAMENTARIAS 2017

\begin{tabular}{lrrrr}
\hline & Mujeres & Hombres & $\begin{array}{r}\text { Diferencia } \\
(p \text {-valor })\end{array}$ & Todos \\
& \multicolumn{1}{c}{$(1)$} & \multicolumn{1}{c}{$(2)$} & \multicolumn{1}{c}{$(3)$} & \\
\hline Indicador para incumbentes & 0,035 & 0,120 & 0,00 & 0,084 \\
Experiencia como legislador & 0,348 & 1,958 & 0,00 & 1,284 \\
Otra experiencia & 0,160 & 0,866 & 0,00 & 0,570 \\
Edad & 44,38 & 48,91 & 0,00 & 47,03 \\
\hline Primero del partido & 0,268 & 0,5 & 0,00 & 0,400 \\
Posición en la lista & 3,789 & 3,275 & 0,00 & 3,490 \\
Posición en el partido & 2,474 & 2,045 & 0,00 & 2,225 \\
Distribución en la lista & 0,652 & 0,558 & 0,00 & 0,597 \\
Distribución en el partido & 0,755 & 0,656 & 0,00 & 0,698 \\
Tamaño de lista & 6,016 & 5,993 & 0,859 & 6,00 \\
Tamaño del partido & 3,548 & 3,62 & 0,515 & 3,498 \\
\hline Aporte del partido (\$) & 3.113 .781 & 4.777 .750 & 0,00 & 4.080 .953 \\
Aporte propio (\$) & 1.252 .188 & 2.948 .235 & 0,00 & 2.238 .005 \\
Aporte de terceros (\$) & 663.439 & 1.632 .457 & 0,00 & 969.018 \\
Aporte total (\$) & 5.029 .408 & 9.358 .441 & 0,00 & 7.545 .634 \\
\hline Total de votos & 5.038 & 8.302 & 0,00 & 6.935 \\
Indicador para ganadores & 0,089 & 0,219 & 0,00 & 0,164 \\
\% votación & 0,022 & 0,037 & 0,00 & 0,030 \\
\hline Número de personas & 451 & 626 & & 1.077 \\
\hline
\end{tabular}

Nota: Las columnas (1) y (2) reportan las medias, la columna (3) reporta el p-valor para el test con hipótesis nula de que la diferencia de medias es igual a cero. El número de observaciones no es igual para todas las variables. La muestra comprende solo los candidatos nominados por los partidos participantes en la elección parlamentaria del 2017.

estudiamos tres mecanismos específicos: (i) pudieron mejorar la posición dentro de la papeleta electoral; (ii) entregar más o menos aportes a algún género, o bien (iii) pudieron asignar a más o menos mujeres a ciertas zonas electorales producto de las características que estas últimas tuvieran, como su tamaño (de manera de aprovechar de mejor forma los incentivos monetarios que enfrentan) o la competitividad del partido en determinado distrito. 


\subsection{Posición de las candidatas en la papeleta electoral}

La cuota puede empujar a los partidos a posicionar a las mujeres en un lugar distinto dentro de la papeleta electoral. Para testear esta hipótesis debemos estimar qué hubiesen hecho los partidos en ausencia de las cuotas. Para lograr este objetivo utilizamos las decisiones de los mismos partidos políticos, pero al momento de tomar las decisiones de posicionamiento de las candidatas en las elecciones de consejeros regionales (cores), elecciones que no estuvieron influidas por la ley de cuotas. Más aún, nuestra metodología compara la diferencia entre la posición de candidatos hombres y mujeres en las elecciones de cores (no afectadas por la ley) con la misma diferencia en las elecciones parlamentarias (sí afectadas por la ley). Además, controlamos por las diferencias entre los tipos de elecciones al usar datos de las elecciones de 2013 y de 2017. De esta manera, nuestra metodología toma en consideración las distintas características de candidatos hombres y mujeres, las diferencias entre tipos de elección, y todos los cambios en el sistema político entre el 2013 y 2017 que afectaron a ambas elecciones de igual manera. ${ }^{8}$

A la hora de calcular si la cuota afectó el posicionamiento de las mujeres utilizamos un marco econométrico. En particular, para testear la hipótesis de interés de manera estadística estimamos la siguiente ecuación:

$$
\begin{aligned}
& \text { posicion }_{\text {ipjzt }}=b_{1}\left(\text { Dip }_{\mathrm{j}}{ }^{*} \text { mujer }_{\mathrm{i}}{ }^{*} 2017_{\mathrm{t}}\right)+\mathrm{b}_{2}\left(\text { Dip }_{\mathrm{j}}{ }^{*} \text { mujer }_{\mathrm{i}}\right)+ \\
& \mathrm{b}_{3}\left(\text { mujer }_{\mathrm{i}}^{* 2017_{\mathrm{t}}}\right)+\mathrm{b}_{4}\left(\text { Dip }_{\mathrm{j}}^{*} 2017_{\mathrm{t}}\right)+\mathrm{n}_{\mathrm{z}}+\mathrm{g}_{\mathrm{p}}+\mathrm{e}_{\mathrm{ipjzt}}
\end{aligned}
$$

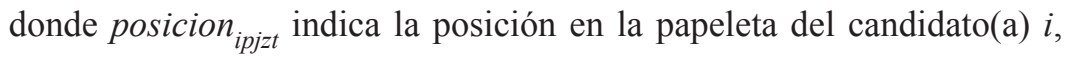
perteneciente al partido $p$, compitiendo en la elección tipo $j$, en la zona electoral $z$, y en el año electoral $t$. Las variables a la derecha se definen como: $\mathrm{Dip}_{j}$ es un indicador que toma el valor de 1 si el candidato(a) está participando en las elecciones parlamentarias y 0 si está participando en las elecciones de consejeros regionales; mujer $_{i}$ es un indicador de género, y 2017 es un indicador que toma el valor de 1 para quienes

8 Vale la pena hacer notar que el uso del posicionamiento de los hombres en 2017 y el posicionamiento de hombres y mujeres en la elección del 2013 tiene como objetivo mejorar la estimación de qué hubiese sucedido el año 2017 en la ausencia de las cuotas. Una simple comparación de hombres y mujeres, o de elecciones parlamentarios con cores, es improbable que entregue una buena estimación. 
compiten en el año 2017 y 0 si lo hacen en el año 2013. Finalmente $n_{z}$ y $g_{p}$ son efectos fijos para las zonas electorales y partidos políticos, que controlan por cualquier diferencia no observable en la estimación en estas dimensiones que no cambie durante el tiempo.

Esta metodología corresponde a diferencias triples en que se presentan dos grupos (cores y parlamentarios), y uno actúa como grupo tratado (parlamentarios) y el otro como grupo de control (cores). Además, se comparan hombres y mujeres antes y después del año del tratamiento. En este caso, el tratamiento está dado por estar afectos a cuotas de género, mientras que la variable de interés estará dada por el posicionamiento de las mujeres en la papeleta electoral. ${ }^{9}$

Las elecciones parlamentarias antes del 2017 se desarrollaban bajo el sistema binominal, en el cual los partidos competían en listas que estaban compuestas por dos candidatos. Esto dificulta la medición de la variable dependiente (posición), porque el nuevo sistema electoral — con el cual se eligen parlamentarios luego de la reforma - permite que las listas nominen a tantos candidatos como escaños a elegir existan en la zona respectiva más un candidato. Para resolver este problema, se ha creado la variable indicador, que toma valor de 1 si el candidato está en la mitad inferior dentro de los candidatos por una lista y 0 si es que se encuentra en la mitad superior. Esta variable ayuda a unificar las dos elecciones con sistemas diferentes. De esta forma, el parámetro de interés corresponde a $b_{1}$ y captura el efecto que tiene ser candidata en las elecciones parlamentarias en el año 2017 según el posicionamiento dentro de la cédula electoral. Este coeficiente captura el efecto causal de la cuota de género en la localización de las mujeres dentro de la papeleta. ${ }^{10}$

${ }^{9}$ En términos técnicos, el supuesto de identificación clave para esta estimación es que el cambio en el posicionamiento de las mujeres candidatas a consejeras regionales en el 2017 es un contrafactual válido para el cambio en el posicionamiento de las mujeres candidatas al parlamento en ausencia de la cuota de género. Es decir, estamos asumiendo que no cambiaron las características de las candidatas al parlamento en el 2017 más de lo que ya diferían con respecto a los hombres y a las mujeres en las elecciones de cores.

${ }^{10}$ Dado que los partidos están obligados a inscribir mujeres en las elecciones parlamentarias, podría suceder que hombres, que sin ley de cuotas hubiesen sido nominados diputados, producto de la cuota pasen a ser nominados consejeros regionales. Esto afectaría el supuesto de identificación de la estrategia empírica utilizada. Sin embargo, lo anterior no parece estar sucediendo, puesto que en ambas elecciones el porcentaje de mujeres nominadas para ser candidatas aumentó. Las candidatas a consejeras regionales pasaron de 19,6 por ciento a 27,1 por ciento entre 2013 y 2017. 
Cuadro 2. POSICIONAMIENTO DE LAS CANDIDATAS EN LA PAPELETA ELECTORAL

\begin{tabular}{lcc}
\hline & $\begin{array}{c}\text { Indicador parte inferior } \\
\text { de papeleta }\end{array}$ & $\begin{array}{c}\text { Indicador para segundo } \\
\text { lugar en papeleta } \\
\text { (submuestra: lugares 1 y 2) } \\
(2)\end{array}$ \\
\hline Parlamentario mujer 2017 & $0,189^{* * *}$ & $0,222^{* *}$ \\
Parlamentario 2017 & $(0,068)$ & $(0,087)$ \\
Parlamentario mujer & $-0,019$ & $-0,033$ \\
Mujer 2017 & $(0,033)$ & $(0,046)$ \\
& $-0,115^{* *}$ & $-0,061$ \\
Observaciones & $(0,054)$ & $(0,054)$ \\
Efecto fijo partido & 0,029 & $0,068^{*}$ \\
Efecto fijo zona electoral & $(0,025)$ & $(0,037)$ \\
\hline
\end{tabular}

Nota: Estas estimaciones utilizan datos a nivel de candidato en las elecciones parlamentarias y de consejeros regionales para los años 2013 y del 2017. En la columna (2) reducimos la muestra a los primeros y segundos candidatos de la lista. Los valores en paréntesis corresponden a errores estándar robustos. Significancia estadistica: ${ }^{* * *}$ $p<0.01,{ }^{* *} p<0.05,{ }^{*} p<0.1$.

La columna 1 del cuadro 2 muestra los resultados de la estimación de la ecuación (1). El coeficiente estimado de interés revela que en las elecciones de 2017 las mujeres aumentaron su probabilidad de estar en la mitad inferior de la papeleta en 18,9 puntos porcentuales. Este resultado constituye evidencia de que los partidos, una vez se vieron más restringidos debido a la ley de cuotas, localizaron a las mujeres en peores posiciones de la papeleta electoral. Como se puede observar en la columna 2 del mismo cuadro, la magnitud del efecto encontrado se mantiene constante aun cuando restringimos la muestra de candidatos a los primeros dos nominados de la lista.

\subsection{Contribución a las campañas políticas}

Para estimar cómo cambia la contribución de los partidos políticos a las campañas electorales de 2017 estimamos la siguiente ecuación por mínimos cuadrados ordinarios: 
$\mathrm{y}_{\mathrm{i}}=\mathrm{a}_{0}+\mathrm{a}_{1}$ mujer $_{\mathrm{i}}+\mathrm{n}_{\mathrm{cp}}+\mathrm{dX} \mathrm{X}_{\mathrm{i}}+\mathrm{u}_{\mathrm{icp}}$

donde $y_{i}$ corresponde a los aportes que recibe el candidato por parte del partido. El parámetro $n_{c p}$ corresponde a un vector de efectos fijos por pacto-zona electoral, mujer ${ }_{i}$ es un indicador que toma valor 1 si la candidata es mujer y 0 si es hombre, y $X_{i}$ es una colección de controles individuales entre los que se incluyen experiencia política (es decir, años como ministro, alcalde o intendente) y edad. El coeficiente de interés es $a_{1}$ y mide cuánto dinero más o menos reciben las mujeres en la campaña electoral del 2017 al compararlas con los hombres.

El cuadro 3 presenta los resultados de la estimación de la ecuación (2), usando como variable dependiente el aporte a los candidatos. Los controles son incorporados paulatinamente entre las diferentes columnas del cuadro. Todas las regresiones controlan por efectos fijos por zona electoral y pacto, si el candidato es incumbente así como también la interacción de esta medida con la variable de género (de manera de diferenciar entre las candidatas que forman ya parte del poder legisla-

\section{Cuadro 3. FINANCIAMIENTO DE CAMPAÑAS DE HOMBRES Y MUJERES}

\begin{tabular}{lcccc}
\hline & $\begin{array}{c}\text { Aporte del partido } \\
(1)\end{array}$ & $\begin{array}{c}\text { pporte propio } \\
(2)\end{array}$ & $\begin{array}{c}\text { Aporte de terceros } \\
(3)\end{array}$ & $\begin{array}{c}\text { Aporte total } \\
(4)\end{array}$ \\
\hline Mujeres & $-0,915^{* * *}$ & $-1,140^{* *}$ & $-1,149^{* * *}$ & $-3,203^{* * *}$ \\
& $(0,322)$ & $(0,443)$ & $(0,378)$ & $(0,728)$ \\
\hline Candidatos & 1.066 & 1.066 & 1.066 & 1.066 \\
Caracteristicas & $\mathrm{X}$ & $\mathrm{X}$ & $\mathrm{X}$ & $\mathrm{X}$ \\
Efecto fijo & & $\mathrm{X}$ & $\mathrm{X}$ & $\mathrm{X}$ \\
pacto-distrito & $\mathrm{X}$ & $\mathrm{X}$ & & \\
\hline
\end{tabular}

Nota: Estimación de corte transversal usando datos a nivel de candidato para las elecciones parlamentarias 2017. Las variables dependientes están medidas en millones de pesos chilenos. "Caracteristicas" incluye si el candidato es incumbente, una interacción entre incumbencia y género, los años de experiencia en el congreso, los años de experiencia en otros cargos de connotación pública, la edad y la edad al cuadrado. Las variables dependientes corresponden a los montos declarados como aportes a las campañas de los candidatos por parte del partido, como aportes a la propia campaña, como aporte de terceros y aporte total. Los candidatos independientes no están incluidos en esta estimación. Errores estándar robustos en paréntesis. ${ }^{* * *} p<0.01,{ }^{* \star} p<0.05$, ${ }^{*} p<0.1$. 
tivo y aquellas que no), la experiencia legislativa y en otros cargos de connotación pública que ha tenido el candidato, la edad y la edad al cuadrado.

Los resultados muestran que los partidos entregan menos recursos a las candidatas mujeres, aun después de controlar por sus características observables. El género está asociado a un cambio de cerca de un millón de pesos en el financiamiento que entregan los partidos a sus candidatos, lo que corresponde al 25 por ciento del total de los aportes recibidos por los aspirantes desde su partido y al 12 por ciento del total del monto de los fondos recibidos para la campaña. El mismo cuadro muestra la diferencia que existe por género para cada una de las fuentes de financiamiento que puedan tener los candidatos para solventar sus campañas. Como es posible observar, para todas las fuentes de financiamiento la variable mujer tiene asociada un coeficiente negativo y estadísticamente significativo.

\subsection{Posicionamiento en territorios electorales}

Un tercer mecanismo que pueden usar los partidos es la ubicación de las candidatas entre las zonas electorales en las que el partido compite. Los partidos tienen dos formas de responder "territorialmente". En primer lugar, dado que los partidos enfrentan diferentes niveles de competitividad en las zonas electorales en las que participan, pueden ubicar a mujeres en circunscripciones y distritos en los que el partido es menos competitivo, reduciendo sus posibilidades reales de obtener un lugar en el Congreso. Y en segundo lugar, los partidos tienen incentivos monetarios a incluir una mayor cantidad de mujeres en zonas electorales de mayor tamaño, de manera de recibir una mayor devolución de los aportes realizados a las candidatas que han nominado, toda vez que esta devolución se realiza según la cantidad de votos totales obtenidos por el candidato y no por el porcentaje de votos obtenidos por estos. ${ }^{11}$

Para testear la presencia de este tercer mecanismo estimamos:

$\%$ mujeres $_{\mathrm{cp}}=\mathrm{b}_{0}+\mathrm{b}_{1} *$ tamaño $_{\mathrm{c}}+\mathrm{b}_{2} *$ competitividad $_{\mathrm{cp}}+\mathrm{s}_{\mathrm{p}}+\mathrm{e}_{\mathrm{cp}}$

${ }^{11}$ Mientras el distrito 27, correspondiente a la región de Aysén, está compuesto por 95 mil electores, el distrito 8, que contiene algunas comunas de la Región Metropolitana como Maipú, supera el millón de electores. 
donde $\%$ mujeres $_{c p}$ corresponde al porcentaje de mujeres que hay en la lista de nominadas por el partido $p$ en la zona $c$, tamaño ${ }_{c}$ corresponde a una medida del tamaño de la zona electoral, competitividad ${ }_{c p}$ es una medida de competitividad por partido que enfrenta en la zona electoral $c$ y $s_{p}$ corresponde a un vector de efectos fijos por partido.

La medida de tamaño utilizada corresponde al logaritmo del número de electores totales con los que cuenta la zona electoral respectiva. En cuanto a la competitividad, la variable ocupada es el menor porcentaje necesario para que el partido deje de tener candidatos electos (en el caso de que los tenga) o pase para elegir algún candidato (y en ese caso esta variable toma valores negativos) conforme a la elección parlamentaria de 2013. En el caso de que un partido no haya competido en la zona electoral respectiva se le ha imputado el valor del partido más cercano ideológicamente. De esta manera se podrá entregar evidencia sobre si los partidos sitúan a las mujeres en lugares en donde estos estiman que poseen menores posibilidades de obtener un buen resultado.

El cuadro 4 muestra los resultados de tres especificaciones de la ecuación (3). Como se puede apreciar, no hay evidencia de que los partidos estén respondiendo al incentivo de tamaño, ya que el porcentaje de mujeres nominadas no varía según el tamaño de las zonas electorales en las cuales el partido participa. Tampoco se observa evidencia de que

Cuadro 4. NOMINACIÓN DE MUJERES POR TAMAÑO Y COMPETITIVIDAD DEL TERRITORIO

\begin{tabular}{lccc}
\hline & \multicolumn{3}{c}{ Porcentaje de candidatas mujeres } \\
\hline Log número electores & $(1)$ & $(2)$ & $(3)$ \\
\hline & 0,036 & & 0,036 \\
Medida de competitividad & $(0,032)$ & & $(0.031)$ \\
& & $-0,012$ & $-0,018$ \\
\hline Observaciones & 430 & $(0,097)$ & $(0,092)$ \\
Efecto fijo partido & $\mathrm{X}$ & 430 & 430 \\
\hline
\end{tabular}

Nota: Cada observación corresponde a una zona-partido en la elección del 2017. La variable de competitividad es el menor porcentaje para que el partido deje de tener candidatos electos en el 2013. En paréntesis presentamos errores estándar robustos. Significancia estadistica: ${ }^{* * *} p<0.01,{ }^{* *} p<0.05,{ }^{*} p<0.1$. 
las mujeres estén postulando en lugares más o menos competitivos. Los signos de los coeficientes implican que a mayor tamaño hay un aumento en la cantidad de mujeres en la lista y que, a un mayor nivel de competitividad, la participación femenina en la lista del partido disminuye. Sin embargo, los efectos no son estadísticamente significativos a niveles convencionales y son de una magnitud relativamente pequeña.

\section{DISCUSIÓN E INTERPRETACIÓN}

¿Por qué los partidos políticos posicionan más abajo en la papeleta a las mujeres y les dan menos dinero para sus campañas? Esta sección evalúa el rol de la cualificación de las candidatas "adicionales" que trajo la ley de cuotas e intenta responder si los resultados se deben a un comportamiento estratégico de los partidos.

\subsection{Selección y cualificación de candidatas}

Dado que existe un cambio en el número de candidatos que participan en las elecciones producto del cambio de sistema electoral entre las elecciones de 2013 y 2017, una potencial explicación para los resultados anteriores sería que se deben a la entrada de candidatos menos cualificados o preparados.

Para responder esta pregunta, se propone seguir la metodología de Besley et al. (2017) y estimar un modelo de diferencias-en-diferencias para el cambio en la brecha entre los ingresos potenciales de hombres y mujeres participantes en las elecciones parlamentarias para los años 2013 y 2017. El objetivo es estimar el cambio en la cualificación de las mujeres en relación con los hombres, de manera de evidenciar si es que la presencia de ventajas al competir para los hombres se podría explicar producto de una mayor cualificación de los hombres con respecto a las mujeres, lo que se debería ver reflejado en mayores diferencias en el ingreso potencial, para el año 2017, entre hombres y mujeres.

Con esta motivación estimamos la siguiente ecuación:

ingreso $_{\text {it }}=b_{0}+b_{1}\left(\right.$ mujer $\left._{i} * 2017_{t}\right)+b_{2}\left(\right.$ mujer $\left._{i}\right)+b_{3}\left(2017_{t}\right)+e_{i t}$

donde ingreso $_{i t}$ corresponde a una medida del potencial de ingresos. Para calcular estos ingresos potenciales usamos una selección aleatoria 
de los candidatos a diputados para los años 2013 y 2017, y recolectamos información acerca de los grados académicos de educación superior (de educación técnica, profesional y de posgrado) completados por cada uno, además de su edad. Luego —ocupando información de ingresos presente en la Encuesta de Caracterización Socioeconómica Nacional 2015 (Casen) - , estimamos el ingreso potencial de los candidatos en el mercado laboral como un proxy de sus habilidades como aspirantes al cargo. Este ingreso potencial se calcula con la edad y educación de los candidatos y candidatas y con los coeficientes estimados de una regresión de Mincer con las siguientes variables: edad, edad ${ }^{2}$ e indicadores para educación técnica, profesional, estudios de postgrado, y para las mujeres. Realizamos la estimación con personas de entre 21 y 79 años, rango de edad de los candidatos y las candidatas en las elecciones. El cuadro 5 reporta estadísticas descriptivas de la información obtenida para estas variables.

Las variables mujer y 2017 en la ecuación (4) corresponden a indicadores de si la candidata es mujer y si compite en el año 2017 (y no en 2013), respectivamente. De esta manera, se busca tener una medida de habilidad de los candidatos y revisar cómo ésta ha variado producto de la incorporación de la cuota. Si la diferencia entre la cualificación

Cuadro 5. EDUCACIÓN DE CANDIDATOS EN ELECCIONES PARLAMENTARIAS 20132017

\begin{tabular}{lrrrr}
\hline & $\begin{array}{c}\text { Mujeres 2013 } \\
(1)\end{array}$ & $\begin{array}{r}\text { Mujeres 2017 } \\
(2)\end{array}$ & $\begin{array}{r}\text { Hombres 2013 } \\
\text { (3) }\end{array}$ & $\begin{array}{r}\text { Hombres 2017 } \\
(4)\end{array}$ \\
\hline Titulo técnico & 2 & 7 & 4 & 8 \\
Titulo profesional & 30 & 31 & 22 & 24 \\
Titulo post-grado & 6 & 8 & 11 & 9 \\
Edad & 41,24 & 44,6 & 49,4 & 48,6 \\
Tngreso (\$) & 1.909 .196 & 1.925 .694 & 2.619 .567 & 2.179 .341 \\
Candidatos & 40 & 48 & 39 & 48 \\
\hline
\end{tabular}

Nota: Número de personas con distintos niveles educativos y edad para una muestra aleatoria de candidatos en las elecciones de 2013 y 2017. Para estimar el ingreso de los candidatos se sigue la metodología de Besley et al. (2017), quienes usan los coeficientes de una regresión de Mincer. En concreto, se utilizaron datos de la encuesta Casen 2015 para personas entre 21 y 79 años y la siguiente estimación: "ingreso_i" = -0.78-0.36*mujer_i+0.47*tecnico_i+1.11*profesional_i+2.12*postgrado_i+0.07*edad_i+-0.01*edad^2_i. Aqui "ingreso_i" es el ingreso estimado de la persona i (en millones de pesos) y todos los coeficientes estimados son estadisticamente significativos (valor-p<0.01) 
Cuadro 6. INGRESO POTENCIAL DE CANDIDATOS LUEGO DE LA LEY DE CUOTAS

\begin{tabular}{lcc}
\hline & \multicolumn{2}{c}{ Ingreso } \\
\hline Mujer 2017 & $(1)$ & $(2)$ \\
& 421.511 & 0,269 \\
Mujer & $(304.279)$ & $(0,180)$ \\
& $-710.371^{* * *}$ & $-0,408^{* * *}$ \\
2017 & $(225.374)$ & $(0,133)$ \\
& $-405.014^{*}$ & $-0,182$ \\
Constante & $(215.903)$ & $(0,127)$ \\
& $2.619 .567^{\star * *}$ & $14.690^{* * *}$ \\
Observaciones & $(160.369)$ & $(0,095)$ \\
\hline
\end{tabular}

Nota: Tal como en el cuadro 5, la metodología para el cálculo de $\widehat{\text { ingreso }}$ sigue la empleada por Besley et al. (2017) y corresponde a una medida del ingreso potencial de los candidatos dada la edad y el nivel educacional de estos, en base a la encuesta Casen 2015 y una regresión de Mircer. Errores estándar en paréntesis. ${ }^{* \star} p<0.01$, ${ }^{* \star} p<0.05$, * $\mathrm{p}<0.1$.

de hombres y mujeres permanece constante en el tiempo ello evidencia, favorablemente, que la cualificación no es uno de los factores que están jugando un rol en el desplazamiento dentro de la papeleta electoral. La cifra de postulantes a diputados pasó de 470, en 2013, a 960 en la elección del 2017. La entrada de un mayor número de candidatos y candidatas podría generar un cambio en su cualificación promedio, lo cual a su vez podría explicar los resultados encontrados en posicionamiento.

El cuadro 6 presenta los resultados. Como se puede apreciar, las mujeres tienen un ingreso potencial menor que los hombres: existe una diferencia estadísticamente significativa de cerca de $\$ 700.000$ en los ingresos potenciales entre géneros. Sin embargo, esta diferencia no se ha visto incrementada entre los años 2013 y 2017; esta brecha se achicó en $\$ 420.000$. Más aún, no podemos rechazar la hipótesis de que esta diferencia se haya reducido para la elección de 2017 (o sea que $b_{1}+b_{2}$ $=0$ ). De esta manera, este resultado, sumado a lo encontrado en la columna 2 del cuadro 2 sugieren que la cualificación entre los candidatos no es una variable que esté explicando los resultados. 


\section{2. ¿Comportamiento estratégico de los partidos políticos?}

Hasta ahora, el comportamiento exhibido se ha señalado como una diferencia en el posicionamiento en la papeleta del voto que despliegan los partidos sobre sus candidatas mujeres. Si bien se ha entregado evidencia a favor de que la educación de los candidatos no estaría jugando un rol en la conducta mostrada por las colectividades, no se le ha asignado una explicación a este patrón de comportamiento.

Una posibilidad es que quienes toman las decisiones de posicionamiento tengan un sesgo implícito en detrimento de las mujeres y que, sin ser conscientes del comportamiento que están desarrollando, posicionan a las mujeres en peores posiciones más allá de las diferencias que son explicadas por la experiencia y la educación de las candidatas. Sin embargo, esto no explica por qué es una estrategia que se inicia una vez que se introduce la cuota de género.

Una explicación consistente con la evidencia encontrada es que la conducta manifestada corresponde a un comportamiento estratégico. Esta hipótesis logra explicar el posicionamiento más abajo en la papeleta después de que se implementa la cuota, puesto que previo a ella el partido podía evitar el uso de este mecanismo dejando de nominar a mujeres en la lista. Además, logra explicar las diferencias en financiamiento que se exhiben, toda vez que es consistente con un agente que ocupa los mecanismos que tiene disponible para entregar ventajas a los hombres por sobre las mujeres.

Una explicación alternativa que no involucra necesariamente un comportamiento estratégico de los partidos está relacionada con potenciales características no observables de las candidatas "adicionales" en las elecciones parlamentarias del 2017, y que no tienen relación con sus ingresos potenciales. Un ejemplo de tal característica es la conexión política de las candidatas. Existe la posibilidad de que al verse necesitados de más candidatas en el año 2017 los partidos tuvieran que recurrir a personas alejadas de las "cúpulas partidistas". Entonces, al llegar la hora de las elecciones las ubicaron en peores posiciones y les dieron menos financiamiento porque no formaban parte de su círculo cercano. Para que esta explicación sea relevante se requiere que los partidos se beneficien más cuando son elegidas las candidatas más cercanas a las cúpulas $y$, además, que estas redes no estén correlacionadas con el ingreso potencial. 


\section{DISEÑOS ALTERNATIVOS DE LAS CUOTAS DE GÉNERO}

A diferencia de leyes de cuotas en otros países, en Chile no fueron desarrollados mecanismos que reglamentaran el posicionamiento de las candidatas en la papeleta electoral. Esto es de particular relevancia, porque, tal como se muestra en Schwindt-Bayer (2009), las cuotas de género exitosas (Suecia o Argentina, por ejemplo) se caracterizan justamente por contar con este tipo de mecanismos. Es por esta razón que resulta importante cuantificar el impacto que ha tenido en el número de parlamentarias electas el hecho de no contar con esta regulación. En particular, evaluamos dos diseños alternativos de la ley de cuotas. Primero, estimamos el cambio en el número de legisladoras si los partidos estuviesen obligados a posicionar a las candidatas en los primeros lugares de la papeleta. El objetivo de esta estimación es obtener una cota superior del número de mujeres que podrían entrar al Congreso si es que aprovecharan al máximo los beneficios del ballot-order effect. Y el segundo escenario evalúa el cambio en el número de parlamentarias electas si aleatorizáramos el orden de género dentro de un mismo partido.

\subsection{Impacto del movimiento de candidatas a los primeros lugares de la papeleta}

Bajo esta primera política alternativa, estimaremos el aumento en el número de mujeres electas si se obliga a los partidos a situar a las mujeres encabezando la lista de nominados. Este contexto representa una cota superior al efecto que pueda tener cualquier política que se implemente a nivel de partidos, pues modifica de la manera más beneficiosa posible la posición de las mujeres. Para calcular el impacto de esta política procedemos de la siguiente forma:

i) Se toman el orden real en el cual los candidatos están puestos para cada partido en cada una de las zonas electorales.

ii) Para cada uno de los partidos y en cada una de las zonas electorales, se separan los candidatos masculinos y femeninos en dos listas distintas. Estas listas mantienen el orden que existía en la lista original para un mismo género. Esto es, si las mujeres del partido estaban en la primera y la cuarta posición dentro de todos los candidatos, en la lista exclusiva de mujeres la primera mujer se mantiene en el primer lugar y quien estaba en el cuarto lugar ahora queda en el segundo. 
iii) Para la creación del nuevo orden contrafactual, se crea la nueva lista a partir de las candidatas tal como se encuentran ordenadas en la lista femenina, seguidas de los candidatos hombres ordenados tal como se encuentran dispuestos en la lista masculina. De esta forma, el "orden contrafactual" de la lista, en el que las mujeres están arriba, queda determinado.

Una vez alterado el orden, se procede a calcular el efecto en votación, el cual se genera a partir de que existe una ganancia en votación al encabezar la lista. Esto lleva a un cambio en el resultado de las votaciones en todas las combinaciones de partido y zona electoral en las cuales la lista no está encabezada por una mujer. En estas combinaciones de zona-partido, el hombre que encabezaba la lista perderá votación, la cual será absorbida por la mujer que lidere el nuevo orden del partido. La magnitud de la ganancia estará dada por el tamaño del ballot-order effect, el cual será escalado por el porcentaje de votación que obtuvo el partido en el distrito o circunscripción respectiva. Asimismo, para alcanzar un panorama más amplio de los efectos que podría tener este fenómeno en el número de mujeres electas $-\mathrm{y}$ dado que la estimación del ballot-order effect en la elección presidencial si bien es informativa, no nos permite determinar de manera precisa el efecto causal de la ganancia en votación como producto de la primacía en la lista partidaria-, se procede a calcular las ganancias en votación para diferentes niveles de ballot-order effect. Una vez que se obtienen los nuevos porcentajes logrados por los candidatos, se procede a calcular el número de mujeres que hubiesen sido electas bajo este nuevo orden.

La figura 3 muestra el número de mujeres adicionales que hubiesen sido electas si es que los partidos hubieran sido forzados a colocar a todas las mujeres en las primeras posiciones. Asimismo, en la figura se muestran, con líneas verticales, los niveles de ballot-order effect estimados para Chile (ver apéndice A).

Vemos, por lo tanto, que el mover a las mujeres a las primeras posiciones tiene un efecto que fluctúa entre dos diputadas adicionales, para la estimación con controles, y cuatro diputadas y una senadora adicional, para la estimación sin controles. Este aumento en representatividad femenina equivale a una reducción de entre 11,1 por ciento $(5,3$ puntos porcentuales) y 22,3 por ciento (10,6 puntos porcentuales) de la 
Figura 3. ESCENARIO CON POSICIONAMIENTO DE CANDIDATAS EN PRIMER LUGAR

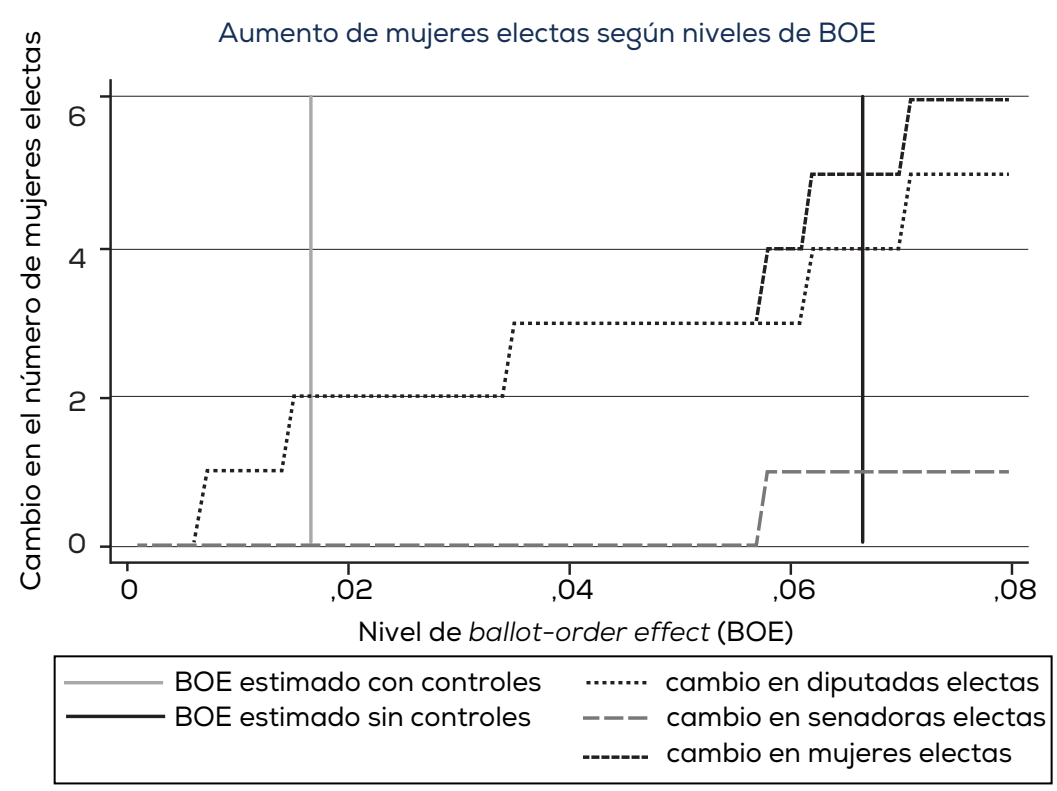

Nota: Este gráfico presenta los resultados de la simulación de una politica en la cual las mujeres ocupan el primer lugar de la papeleta electoral dentro de cada uno de los partidos. El eje $y$ mide el aumento de mujeres electas $y$ el eje $x$ mide los distintos niveles de ballot order eect (BOE) encontrados en la literatura. Las líneas verticales denotan nuestras estimaciones del BOE para el caso chileno.

brecha que separa a Chile de los países que más han tenido efectividad a la hora de incrementar la participación femenina con las cuotas de género. ${ }^{12}$

\subsection{Impacto de un orden aleatorio}

Si bien el escenario anterior es el que maximiza las oportunidades de las mujeres dentro de las elecciones para la obtención de los escaños que están en disputa, el mover a las mujeres hacia arriba puede consi-

12 Para esta brecha se utilizaron únicamente los diputados, por lo que la ganancia en una senadora adicional para el caso superior no es tomado en consideración para estos efectos. 
derarse como una forma de discriminación arbitraria, en cuanto asigna beneficios a un grupo producto de las características que éste tiene. Por esta razón, y en vista a disminuir todo tipo de arbitrariedad existente en la nominación de los candidatos, estimamos el cambio en el número de mujeres electas al aleatorizar el orden de los candidatos en las listas de los partidos.

A diferencia del caso anterior, en este escenario el aumento de los escaños obtenidos por las aspirantes mujeres no está asegurado, puesto que así como mujeres pueden mejorar su posición, también habrá candidatas que empeorarán la localización dentro del voto en la que se encuentran. No obstante, como se ha estimado en las secciones anteriores, las mujeres tienen a priori una probabilidad menor de encabezar las listas de nominados por los partidos, por lo que es esperable que este resultado se vea corregido en favor de las mujeres.

Para la estimación generamos 100 órdenes aleatorios de los candidatos a nivel de partido-zona electoral. Luego procedemos a modificar repetidas veces la votación obtenida por los candidatos usando ballotorder effects de 0,1 a 8,0 puntos porcentuales. Luego se determina, para cada una de las simulaciones, en cada uno de los ballot-order effects, cuáles son los candidatos elegidos y cuántos de estos candidatos corresponden a mujeres. Una vez conocido el número de mujeres electas, se procede a obtener la media de parlamentarias y diputadas ganadoras y los percentiles 5 y 95 de la distribución observada de parlamentarias y diputadas escogidas.

La figura 4 presenta los resultados. Este gráfico muestra que para la estimación con controles el número de mujeres en el parlamento aumenta en 1,5, y en el mismo número para el caso de las diputadas. Esto equivale a una reducción de 9,1 por ciento (4 puntos porcentuales) en la brecha que separa a Chile de los países con cuotas implementadas más efectivas. Del mismo modo, con la estimación del ballot-order effect sin controles el número de parlamentarias electas aumenta en 1,27, mientras que el número de diputadas lo hace en 0,72 (1,9 puntos porcentuales), esto equivale a una reducción del 4,2 por ciento de la brecha.

A partir de los impactos calculados, estimamos que con una política de mujeres en los primeros lugares de la papeleta Chile lograría alcanzar a Colombia en cuanto a efectividad de sus cuotas, mientras 
Figura 4. ESCENARIO CON POSICIONAMIENTO ALEATORIO DE CANDIDATAS

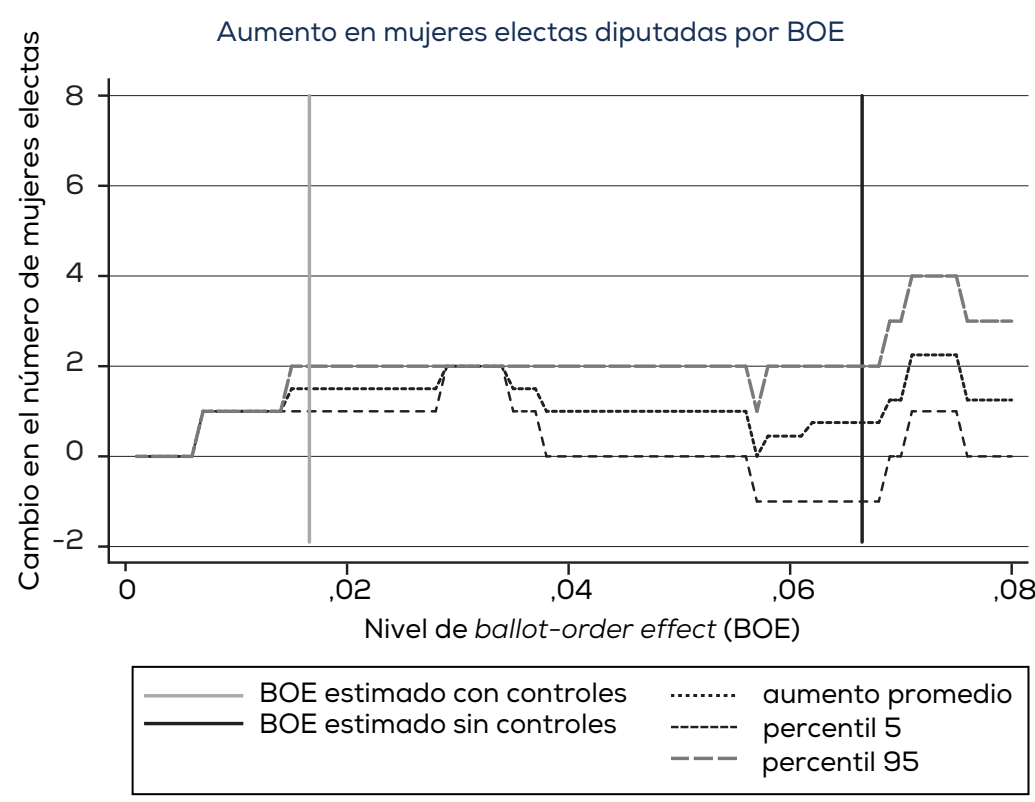

Nota: Este gráfico presenta los resultados de la simulación de una política en la cual las mujeres ocupan un lugar aleatorio dentro de la papeleta electoral de cada uno de los partidos. El eje $y$ mide el aumento de mujeres electas $y$ el eje $x$ mide los distintos niveles de ballot order eect (BOE) encontrados en la literatura. Las lineas verticales denotan nuestras estimaciones del BOE para el caso chileno.

que para los casos aleatorios el cambio obtenido respecto de la situación inicial es más modesto. De este modo, podemos afirmar que, si bien el peor posicionamiento femenino por parte de los partidos está jugando un rol a la hora de aumentar la participación de las mujeres en el parlamento, éste no logra explicar más del 20 por ciento del total de la brecha que existe en efectividad de la implementación de la cuota entre Chile y las naciones que más han tenido éxito en este sentido. ${ }^{13}$

${ }^{13} \mathrm{La}$ diferencia puede corresponder al efecto que encontramos en gasto electoral, pero, a diferencia de lo que implica el ballot-order effect, no tenemos estimaciones que nos permitan documentar el efecto en votación de las diferencias de gasto. 


\subsection{Limitaciones al análisis de políticas alternativas}

Para una mejor interpretación de los resultados entregados, se deben tomar en consideración las limitaciones que estos tienen a la hora de evaluar posibles políticas públicas alternativas que afecten el ordenamiento de los candidatos dentro del voto.

El análisis recién desarrollado supone que los partidos se comportan de la misma manera en que se hubiesen comportado de manera previa a las limitaciones propuestas al posicionamiento en la papeleta. Pero el comportamiento de los partidos es dinámico y puede modificarse al cambiar las reglas del juego.

Las políticas alternativas propuestas disminuyen los grados de libertad que los partidos poseen al posicionar a sus candidatos dentro de la papeleta electoral. En cualquiera de las propuestas, las colectividades ya no pueden elegir poner a los hombres por sobre mujeres. Sin embargo, como se ha documentado en este trabajo, el posicionamiento dentro de la papeleta electoral no es el único mecanismo que los partidos han ocupado para tratar en forma diferente a candidatos y candidatas. Al ver restringido el canal de posicionamiento dentro del voto, los partidos podrían comenzar a utilizar con más intensidad alguno de los otros dos canales mencionados: el aporte en dinero a las candidatas o su ubicación en zonas electorales donde el partido es menos competitivo o de mayor tamaño.

Una segunda consideración a tomar en cuenta tiene que ver con la cualificación de las mujeres puestas a competir en las zonas electorales en las que participan. En las políticas alternativas propuestas, la probabilidad de que la lista la encabece un hombre se reduce cuando el orden es aleatorio, o es igual a cero cuando se les obliga a que las mujeres encabecen las listas. En consecuencia, ello puede convertirse en un incentivo a buscar mujeres más cualificadas o a aumentar la cualificación de las posibles candidatas. Por lo tanto, un posible efecto colateral que tendría este tipo de política es positivo, ya que empujaría a aumentar la cualificación para las mujeres, dado que los partidos ya no podrían controlar quién será su candidato beneficiado por el ballotorder effect. 


\section{CONCLUSIÓN}

Este artículo examina las estrategias de nominación de los partidos para las elecciones parlamentarias chilenas del año 2017 a efectos de explicar el relativo bajo impacto de la ley de cuotas. Los resultados muestran que los partidos posicionan a las candidatas en lugares más bajos dentro de la papeleta electoral - lo cual no es producto de la entrada de candidatas menos cualificadas_- que las mujeres reciben menores montos de dinero como aportes desde su partido y que los partidos no responden al incentivo monetario que existe para las candidatas en las devoluciones de gastos de campañas.

El aporte de este artículo radica, en primer lugar, en ampliar el estudio de los efectos que tiene la incorporación de cuotas de género, particularmente en contextos fuera de Europa en donde se ha realizado el grueso del estudio de este tipo de políticas, enfatizando la importancia de un potencial comportamiento estratégico de los partidos políticos que podría impedir el correcto funcionamiento de las cuotas. En segundo lugar, éste es el primer trabajo que intenta estimar el efecto que tendrían políticas alternativas - posibles de implementar- en el número final de mujeres electas, lo cual amplía el análisis económico que se ha hecho en la literatura respecto a los efectos que las cuotas han acarreado.

\section{APÉNDICE}

\section{A. Estimación del ballot-order effect (BOE) para Chile}

Para la estimación del ballot-order effect usamos información del Servel consistente en: resultados, número de participantes en la elección y el orden de los candidatos para las elecciones presidenciales entre los años 1989 y 2017. Se cuenta con esta información a nivel de candidato, por lo que se tienen 50 observaciones para las 10 votaciones en las que participó un total de 40 candidatos durante el periodo estudiado. Para estas elecciones, el orden de los candidatos dentro de la cédula electoral es sorteado públicamente. También contamos con información de características observables de los candidatos: porcentaje estimado por el Centro de Estudios Públicos (CEP) de votación para 
cada candidato presidencial en la última encuesta anterior a la elección correspondiente, ${ }^{14}$ el género del candidato, la distribución ideológica del aspirante (izquierda, centro o derecha) y si es que éste forma parte de las dos grandes coaliciones que han gobernado el país (Alianza y Concertación). El cuadro A.1 muestra el promedio de los observables por lugar en la papeleta electoral (primero corresponde a un indicador que toma valor 1 si es que el candidato es el primer nombre dentro de la papeleta electoral y 0 si es que se encuentra en las posiciones restantes). Como se puede apreciar $-\mathrm{y}$ tal como es esperable dada la aleatoriedad del sorteo-, las variables observables no tienen, en la mayoría de los casos, diferencias significativas según la posición de los candidatos. No obstante, se tomarán en consideración dentro de las estimaciones que se hagan para el ballot-order effect con el objetivo de aumentar la precisión de los estimadores obtenidos en las regresiones.

Para la estimación explotaremos el orden aleatorio de los candidatos en las elecciones presidenciales. Siguiendo a Miller y Krosnick (1998) estimamos:

$\%$ votos $_{\text {iet }}=\mathrm{d}_{0}+\mathrm{d}_{1}$ orden $_{\text {iet }}+\mathrm{gX}_{\mathrm{i}}+\mathrm{e}_{\text {ict }}$

donde por ciento votos ${ }_{i e t}$ corresponde al porcentaje de votación del candidato $i$ en la elección $e$ en el año $t$; orden $_{i e t}$ corresponde a la medidas orden, en donde siguiendo la literatura existente acerca de ballot-order effect nos centraremos en la ganancia de votación al ser nominado como primer candidato del partido, y $X_{i}$ corresponde a un vector de características observables de los candidatos. Dado que por la aleatoriedad del sorteo el error y la posición no están correlacionados entre sí, se estará capturando el efecto causal de la posición en el porcentaje de votos obtenido.

Sin embargo, tal como se discute en diversos trabajos relacionados (Miller y Krosnick 1998; Ho e Imai 2008; King y Leigh 2009), el efecto en votación que tiene la posición en el voto depende del nivel de información que tengan los votantes a la hora de manifestar sus preferencias. De este modo — dada la importancia y la cantidad de información disponible en los distintos medios de comunicación-, la estimación

${ }^{14}$ Esta encuesta, a su vez, es tomada previo a que el orden en el voto de los candidatos sea determinado a través del sorteo realizado por el Servel. 
obtenida se debe tomar como una cota inferior del ballot-order effect existente para las elecciones parlamentarias.

El cuadro A.2 presenta los resultados. Las columnas 1 a 3 utilizan como variable dependiente el porcentaje de votación obtenido por el candidato en la elección mientras que las columnas 4 a 6 tienen como resultado el logaritmo del porcentaje obtenido en los comicios. Las columnas 1 y 4 no utilizan controles, en tanto que las columnas 2 y 5 incluyen en la estimación el control de la última encuesta CEP antes de la elección presidencial respectiva, debido a que es la principal variable explicativa del porcentaje obtenido por el candidato. Finalmente, las columnas 3 y 6 incorporan al resto de características observables que se presentan en el cuadro A.1.

Como se puede apreciar, el indicador para estar primero dentro del voto no resulta estadísticamente significativo en ninguna de las especificaciones reportadas para ningún nivel de significancia relevante. Sin embargo, el tamaño de los efectos es grande y está en línea con las magnitudes que se encuentran en la literatura (entre 1 y 6 puntos porcentuales). Esto se debe al bajo número de observaciones con los que se cuenta para realizar la estimación. De esta manera, no podemos contar con una estimación certera de cuál es el porcentaje de ganancia que reciben los candidatos al ser seleccionados en el primer lugar. No obstante, el estimador punto sí nos sirve para obtener una referencia acerca de los valores en los que se puede encontrar el ballot-order effect para el caso chileno. 
Cuadro A.1. CARACTERÍSTICAS DE CANDIDATOS EN LAS ELECCIONES PRESIDENCIALES 1989-2013 POR POSICIÓN EN LA PAPELETA ELECTORAL

\begin{tabular}{lrrrrrr}
\hline & Primero & Resto & Diferencia & $p$-valor & $\begin{array}{r}p \text {-valor } \\
\text { permut. }\end{array}$ & Todos \\
& \multicolumn{1}{c}{$(1)$} & \multicolumn{1}{c}{$(2)$} & \multicolumn{1}{c}{$(3)$} & \multicolumn{1}{c}{$(4)$} & \multicolumn{1}{c}{$(5)$} & \multicolumn{1}{c}{$(6)$} \\
\hline Pronóstico CEP & 0,211 & 0,189 & 0,023 & 0,71 & 0,68 & 0,194 \\
Mujer & 0,167 & 0,237 & $-0,07$ & 0,62 & 0,65 & 0,22 \\
Años educación & 20,33 & 18,37 & 1,96 & 0,02 & 0,03 & 18,84 \\
Experiencia politica & 6,5 & 6,08 & 0,42 & 0,83 & 1,00 & 6,18 \\
Ex-presidente & 0,167 & 0,105 & 0,061 & 0,58 & 0,67 & 0,159 \\
Centro & 0,25 & 0,184 & 0,066 & 0,63 & 0,73 & 0,2 \\
Izquierda & 0,333 & 0,526 & 0,193 & $-0,25$ & 0,48 & 0,48 \\
Derecha & 0,417 & 0,289 & 0,127 & 0,42 & 0,47 & 0,32 \\
Alianza & 0,417 & 0,211 & 0,206 & 0,16 & 0,29 & 0,26 \\
Concertación & 0,25 & 0,263 & $-0,013$ & 0,93 & 1,00 & 0,26 \\
\hline Total votos & 1.957 .920 & 1.532 .972 & 424.948 & 0,37 & 0.96 & 1.634 .960 \\
\% votos & 0,284 & 0,218 & 0,067 & 0,33 & 0,86 & 0,234 \\
Electo & 0,333 & 0,211 & 0,123 & 0,40 & 0,36 & 0,24 \\
\hline Observaciones & 12 & 38 & & & & 50 \\
\hline
\end{tabular}

Nota: Cada observación corresponde a un candidato-elección. Las columnas 1-2 presentan los promedios de variables para candidatos en el primer lugar de la papeleta y el resto. Las columnas 3-4 reportan el p-valor para el test con hipótesis nula de que la diferencia de medias es igual a cero, la columna 3 es un test de medias simple y la columna 4 ajusta por el tamaño de muestra relativamente pequeño.

Cuadro A.2. ESTIMACIÓN DEL BALLOT ORDER EECT USANDO ELECCIONES PRESIDENCIALES

\begin{tabular}{lccrrrrr}
\hline & \multicolumn{3}{c}{$\%$ votos } & \multicolumn{3}{c}{ log \% votos } \\
\hline & $(1)$ & $(2)$ & $(3)$ & $(4)$ & $(5)$ & $(6)$ \\
\hline Indicador primero en la papeleta & 0,062 & 0,017 & 0,008 & 0,516 & 0,273 & 0,218 \\
& $(0,067)$ & $(0,017)$ & $(0,018)$ & $(0,563)$ & $(0,344)$ & $(0.349)$ \\
\hline Control última encuesta CEP & & $\mathrm{X}$ & $\mathrm{X}$ & & $\mathrm{X}$ & $\mathrm{X}$ \\
Otros controles & & & $\mathrm{X}$ & & & $\mathrm{X}$ \\
\hline Observaciones & 50 & 48 & 48 & 50 & 48 & 48 \\
R-cuadrado & 0,02 & 0,94 & 0,95 & 0,02 & 0,67 & 0,77 \\
\hline
\end{tabular}

Nota: Las estimaciones cuentan con ponderaciones por el número de personas que participaron en la elección correspondiente. "Otros controles" incluyen el género del candidato, si es que ha sido ex-presidente, indicadores por tendencia política, un indicador para los dos grandes conglomerados politicos (Alianza y Concertación) y el número de años de educación de los candidatos. Errores estándar robustos en paréntesis. Significancia estadistica: ${ }^{* \star}{ }^{* *}<0.01,{ }^{* *} p<0.05,{ }^{*} p<0.1$. 


\section{REFERENCIAS}

Bagues, M. \& P. Campa. 2017. Can gender quotas in candidate lists empower women? Evidence from a regression discontinuity design. Manuscrito.

Baltrunaite, A., P. Bello, A. Casarico \& P. Profeta. 2014. "Gender quotes and the quality of politicians". Journal of Public Economics 118: 62-74.

Beaman, L., R. Chattopadhyay, E. Duflo, R. Pande \& P. Topalova. 2009. "Powerful women: Female leadership and gender bias". Quarterly Journal of Economics 124 (4): 1497-1540.

Besley, T. J., O. Folke, T. Persson \& J. Rickne. 2017. "Gender quotes and the crisis of the mediocre man: Theory and evidence from Sweden". American Economic Review 107 (8): 2204-2242.

Caminotti, M. \& F. Freidenberg. 2016. "Federalismo electoral, fortaleza de las cuotas de género y representación política de las mujeres en los ámbitos subnacionales en Argentina y México". Revista Mexicana de Ciencias Políticas y Sociales 228: 121-144.

Clots-Figueras, I. 2011. "Women in politics: Evidence from the Indian States". Journal of Public Economics 95: 664-690.

De Paola, M., V. Scoppa \& R. Lombardo. 2010. "Can gender quotas break down negative stereotypes? Evidence from changes in electoral rules". Journal of Public Economics 94 (5): 344-353.

Economist Intelligence Unit. 2017. The Democracy Index. https://infographics. economist.com/2017/DemocracyIndex/. Último acceso el 27 noviembre, 2017.

Esteve-Volart, B. \& M. Bagues. 2012. "Are women pawns in the political game? Evidence from elections to the Spanish Senate". Journal of Public Economics 96 (3): 387-399.

Frechette, G., F. Maniquet \& M. Morelli. 2008. "Incumbents interests and gender quotes". American Journal of Political Science 52 (4): 891-909.

Freedom House. 2017. "Freedom in the World". https://freedomhouse.org/report/ freedom-world/2017/chile. Último acceso el 27 noviembre, 2017.

Gold, D. 1952. "A note on 'rationality' of anthropologists in voting for officers". American Sociological Review 17: 99-101.

Gulzar, S. \& N. Ruiz. 2018. "Ballot order effects and party respondes: Evidence from Lotteries in Colombia". Judgment and Decision Making 6 (4): 333-342.

Ho, D. \& K. Imai. 2006. "Randomization inference with natural experiments: An analysis of ballot effects in the 2003 California recall election". Journal of the American Statistical Association 101 (475): 888-900.

_. 2008. "Estimating causal effects of ballot order from a randomized natural experiment: The California alphabet lottery, 1978-2002". Public Opinion Quarterly 72 (2): 216-240.

International IDEA, Inter Parliamentary Union \& Stockholm University. 2018. Gender Quotas Database. 
Kanthak, K. \& J. Woon. 2015. "Women don't run? Election aversion and candidate entry". American Journal of Political Science 59 (3): 595-612.

King, A. \& A. Leigh. 2009. "Are ballot order effects heterogeneous?" Social Science Quarterly 90 (1): 71-87.

Koppell, J. \& J. Steen. 2004. "The effects of ballot position on election outcomes". Journal of Politics 66 (1): 267-281.

Miller, J. \& J. Krosnick. 1998. "The impact of candidate name order on election outcomes". Public Opinion Quarterly 62 (3): 291-330.

Miranda, L. \& J. Suárez-Cao. 2018. La política siempre ha sido cosa de mujeres: elecciones y protagonistas en Chile y la Región. Santiago: Ediciones Flacso Chile.

Schwindt-Bayer, K. 2009. "Making quotas work: The effect of gender quota laws on the election of women". Legislative Studies Quarterly 34 (1): 5-28.

Schwindt-Bayer, K. (editor). 2018. Gender and Representation in Latin America. Nueva York: Oxford University Press. EP 